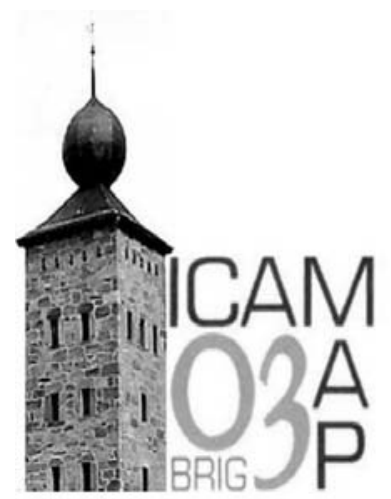

\title{
International Conference on Alpine Meteorology and MAP Meeting 19-23 May 2003, Brig, Switzerland
}

\author{
Andrea RosSA $^{* 1}$ (Guest Editor), CORnElia SchwiERZ ${ }^{2}$ (Guest Editor) and MARKUS FurGER ${ }^{3}$ \\ ${ }^{1}$ MeteoSwiss, Zürich, Switzerland \\ ${ }^{2}$ Institute for Atmospheric and Climate Science, ETH, Zürich, Switzerland \\ ${ }^{3}$ Paul Scherrer Institut, Laboratory of Atmospheric Chemistry, Villigen, Switzerland
}

It is with pleasure that we present the second part of the three-part ICAM/MAP Special Issue of the Meteorologische Zeitschrift. As outlined in the first part's editorial, this conference was the first joint edition of the traditional International Conference on Alpine Meteorology (ICAM) and the annual scientific meeting of the Mesoscale Alpine Programme (MAP). Here we take a moment to describe MAP along general lines. MAP is a concerted research effort of the international meteorological and hydrological communities to further the understanding and prediction of intense weather in mountainous areas, and as such the largest of its kind in the European Alps since ALPEX, the Alpine Experiment, conducted in 1982.

On a historical note, the first idea for MAP was that of a Foehn project and emerged within the ALPEX community in the early nineties. Its final concept, however, was forged during a workshop in Zurich in September 1994, where the necessity to include heavy precipitation into the core scientific targets was recognized. By 1996 hydrology became another important element in MAP. In light of the unhappily regular series of autumnal flooding events in different parts of the Alps throughout the last decade, these decisions proved to be essential and well taken. The goals of MAP are laid out in the Design Proposal and detailed in the Science Plan, to encompass 'wet' and 'dry' MAP objectives, short hand for orographically induced precipitation including hydrological runoff, and three-dimensional circulation patterns in the vicinity of large mountain ranges. An important supportive objective of MAP is to provide an observational basis for the validation of high-resolution numerical weather prediction models over complex terrain, thus strengthening the link between research and application. Both the Design Proposal and the Science Plan are available online at www.map.ethz.

The overall structure of the programme is divided into three distinct phases that were and are managed by three bodies, which took care of the political, scientific, and practical issues, and the permanent Programme Office and MAP Data Centre. The latter, funded by an optional EUMETNET ${ }^{1}$ Programme, proved to be absolutely vital for the success of MAP. The Special Observing Period (SOP) of MAP, the actual Field Phase, took place 7 September - 15 November 1999 and was preceded by an extensive and careful Planning Phase. The high scientific level of the

\footnotetext{
*Correspondence address: Andrea Rossa, MeteoSchweiz, Krähbühlstrasse 58, CH-8044 Zürich, Schweiz,

e-mail: andrea.rossa@meteoschweiz.ch

${ }^{1}$ EUMETNET, the European Meteorological Network, is a network grouping 17 European National Meteorological Services, to provide a framework to organise co-operative programmes between the Members in various fields, such as observing systems, data processing, basic forecasting products, research and development, and training.
} 
conference, to which this Special Issue is dedicated, bears witness to an extremely productive Evaluation Phase, the third phase of MAP.

The dissemination of the MAP results gathered so far is ongoing. At the ICAM/MAP 2003 an overview presentation on the main results obtained up to now was given, reflecting the content of a special issue of the Quarterly Journal of the Royal Meteorological Society. It appeared January 2003 and is to be followed by a volume of the Journal on Hydrology and Earth System Sciences (HESS) and the present issues of the Meteorologische Zeitschrift. Besides these, a large number of MAP papers have been published in a variety of journals. A comprehensive list of MAP-related publications can be found at the MAP Data Centre (www.map.ethz.ch). Of the many papers presented at the conference and in this Special Issue we would like to mention two at this stage, while more will be said about them in the third and final volume. In this volume, HÄBERLI et al. report on a EUMETNET-funded data quality control exercise in which the performance of the surface observational networks available during MAP, one of the densest in the world, was assessed. Motivated by lessons learnt during ALPEX, this effort is particularly relevant in view of validating the numerous studies performed with high-resolution numerical models. Secondly, in part one of this Special Issue PARKER presents and confronts MAP with the perspective of the societal needs when it comes to improving flood forecasting, warning and response systems.

As a matter of fact, the WMO World Weather Research Programme (WWRP), of which MAP was the first research initiative WWRP endorsed, strongly encourages research programmes to demonstrate the societal impact of their endeavours. WWRP devised the concept of a Forecast Demonstration Project (FDP) which is geared to achieving exactly this. It involves forecasting of high-impact weather of international relevance with clear evaluation protocols that will document advances in operational forecasting. At present a MAP working group is exploring the possibility to conduct such an FDP in order to demonstrate the societal impact of MAP. This would then be the fourth phase of MAP.

The ICAM/MAP Meeting 2003 was organized by MeteoSwiss and the Institute for Atmospheric and Climate Science of ETH Zurich (IACETH), and co-sponsored by the Swiss Society for Meteorology (SGM), the European Meteorological Society (EMS), and the American Meteorological Society (AMS). 